Prof. Martin Halle,

Lehrstuhl für präventive und rehabilitative Sportmedizin, Zentrum für Prävention \& Sportmedizin der TU München

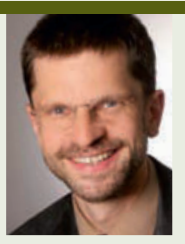

Prof. Ulf Müller-Ladner, Direktor der Abteilung Rheumatologie und Klinische Immunologie, Kerckhoff-Klinik gGmbH,

Bad Nauheim

\section{Das sagt der Kardiologe}

Zu Frage 1: Wir befinden uns hier in einem Dilemma: Auf der einen Seite haben wir Kenntnis von dem kardiovaskulären Risiko, auf der anderen Seite haben wir Patienten mit akuten Schmerzen. Die meisten Arthrosepatienten haben schon aufgrund ihres Alters kardiovaskuläre Risikofaktoren. Etwa 40\% der ab 60-Jährigen leiden an einer arteriellen Hypertonie.

Wenn ich längerfristig NSAR einsetze, muss ich also das kardiovaskuläre Risikoprofil der Patienten optimieren. Denn man wird nicht um NSAR herumkommen - zumal sie das Risiko deutlich weniger erhöhen als etwa eine Hypertonie oder eine Dyslipidämie. Die Verordnung von NSAR kann auch eine Chance sein, einen Patienten zu einer besseren Kontrolle von kardiovaskulären Risikofaktoren zu motivieren. Indem man ihm zum Beispiel sagt, wenn er nicht mit dem Rauchen aufhört, kann er dieses Medikament nicht bekommen, weil er sonst einen Herzinfarkt erleiden kann. Die Dosierung spielt dabei eine untergeordnete Rolle. Es geht um Verordnung ja oder nein.

zu Frage 2: Nein, dieses Risiko ist nur bei einer Langzeittherapie relevant. Auch bei Postinfarktpatienten sehe ich nach einem Intervall von vier Wochen und ausreichender antithrombotischer Therapie keine Kontraindikation. Die Evidenz hierfür ist allerdings schwach.

zu Frage 3: Man kann Opioide geben, aber dann hat man das Problem der Obstipation. Paracetamol ist wesentlich schwächer wirksam und in höheren Dosen hepatotoxisch, das ist keine realistische Alternative. Geeigneter ist eine Kombination aus einem niedrig dosierten Coxib und Paracetamol, um Schmerzspitzen abzudecken. Durch eine komplette Umstellung fängt man sich negative Effekte ein.

Ich denke aber, dass NSAR zu großzügig verordnet werden. Bei Arthrosepatienten geht es darum, den Akutschmerz zu nehmen. Dafür kann man das Medikament am Anfang auch ein paar Wochen regelmäßig einnehmen. Aber danach sollte eine Bedarfstherapie stattfinden, und vor allem sollten andere Optionen wie die Physiotherapie stärker ausgeschöpft werden. Zum Beispiel kann die Kräftigung der Oberschenkelmuskulatur nachweislich Schmerzen reduzieren.

\section{Das sagt der Rheumatologe}

zu Frage 1: Wie bei jedem internistischen Patienten müssen grundsätzlich alle Organe und Organsysteme berücksichtigt werden. Das heißt, das kardiovaskuläre Risiko muss genauso entsprechend den Empfehlungen behandelt werden wie die rheumatische Erkrankung. Und für alle Medikamente gilt: nur so viel wie nötig. Aber wenn ein Patient NSAR braucht, um sich wieder schmerzfrei bewegen zu können und damit sein kardiovaskuläres Risiko zu minimieren und den schmerzbedingten Stress zu reduzieren, gibt es keinen Grund, auf NSAR zu verzichten. Wenn bei einem Patienten ein potenzielles kardiovaskuläres Risiko besteht, muss parallel eine kardiologische Vorstellung erfolgen. Die weitere Überwachung dieses Risikos ist Teil der regulären internistisch-rheumatologischen Kontrolle.

zu Frage 2: Es gibt keine Evidenz für eine Erhöhung des kardiovaskulären Risikos unter einer kurzfristigen bzw. intermittierenden Therapie. Nur in den ersten Wochen nach einem frischen Herzinfarkt sollte vermieden werden, ein NSAR einzusetzen. Bei ausgeprägten Gelenkschmerzen muss aber teilweise doch darauf zurückgegriffen werden.

zu Frage 3: Paracetamol ist ein reines Schmerzmittel und als NSAR-Ersatz vor allem bei der rheumatoiden Arthritis nicht geeignet. Auch Opioide sind in der Therapie entzündlicher Gelenkerkrankungen eine reine Reservemedikation. Entsprechende Studien ergaben keinen wirklichen Nutzen bezüglich der Grunderkrankung. Ob Paracetamol und Opioide in kardiovaskulärer Hinsicht günstiger sind als NSAR, ist nicht erwiesen. Mit neuen Biologika sind mögliche Alternativen in Sicht, aber sie befinden sich noch in der klinischen Prüfung. 